\title{
NON-UNIFORM EXTENSION OF A NON-LINEAR VISCOELASTIC SLAB
}

\author{
Fuling Dal and K. R. Rajagopal \\ Department of Mechanical Engineering, University of Pittsburgh, Pittsburgh. PA 15261, U.S.A. \\ and \\ A. S. WINEMA: \\ Department of Mechanical Engineering and Applied Mechanics, University of Michigan, \\ Ann Arbor. MI 48109. U.S.A.
}

(Receited 21 August 1990 ; in recised form 8 Julv 1991)

\begin{abstract}
The non-uniform extension of a slab of a non-linear viscoelastic solid is studied. Two boundary value problems are solved. one with deformation prescribed at the upper boundary and the other with the traction prescribed. The formulation uses the deformation in the direction of the slab thickness as the dependent variable, which is found by solving a non-linear integro-differential equation. The numerical calculation is such that at each time step, the problem is equivalent to a fourth order non-linear ordinary differential equation for the current coordinate in the direction of the slab thickness. This equation is then integrated by the same numerical procedure as in the corresponding elastic problem.
\end{abstract}

\section{INTRODUCTION}

Few initial-boundary value problems have been solved within the context of genuinely nonlinear viscoelastic solids. Wincman (1972, 1978) studied the response of non-lincar axially symmetric viscoelastic membranes and found the possibility of multiplicity of solutions. However, this study which prognosticated interesting possibilities with regard to the bifurcation and stability of problems involving finite deformations of highly non-linear viscoelastic solids has never been followed up with the kind of serutiny the area deserved. In this work. we provide a rather interesting analysis involving the non-homogeneous extension of a non-lincar viscoclastic slab.

The problem under consideration can be considered as having arisen from another train of thought. In recent years there has been al considerable amount of interest in the study of non-homogeneous deformations in non-linearly elastic solids [cf. Currie and Hayes (1982), Rajagopal and Wineman (1985), Rajagopal et al. (1986)]. In view of Ericksen's results $(1954,1955)$ that in an isotropic compressible elastic material "universal solutions" are homogeneous, not much work has been expended in studying non-homogeneous deformations as they are not possible in all isotropic compressible materials. However, since non-homogeneous deformations are the order of the day, recently, attention has turned to seeking an answer to the following question: given a non-homogeneous deformation, can we determine the largest (or at least a large enough) class of constitutive equations that can support such a non-homogencous deformation?

Rajagopal and Wineman (1985) showed that for a non-linear elastic slab a class of non-uniform uniaxial extensions (or compressions) were possible within the context of the Mooney-Rivlin theory. They find that the classical uniaxial solution corresponds to the special case which corresponds to a specific structure for the pressure field, namely it being a constant. In this study we investigate whether such non-homogeneous solutions are possible within the context of non-linear viscoelasticity. In this case the problem turns out to be even more interesting for there are two possible problems, one in which the displacement of the slab is specified and held fixed, and the other in which the appropriate traction is prescribed and held constant. While Rajagopal and Wineman (1985) were able to obtain exact closed form solutions in the case of a Mooncy-Rivlin material, the equations governing the viscoelastic problems are too complicated to be amenable to such an analysis and have to be solved numerically.

We consider the elongation of a slab of thickness $H$, its boundaries defined by the planes $Z=0$ and $Z=H$. It is found that when the viscoelastic slab is subject to a step 
elongation. an inhomogeneous deformation is possible and the stresses in the slab vary inhomogeneously and "relax" with time. Inhomogeneous deformations are also possible when tractions are prescribed on the boundaries. When tractions are prescribed on the boundaries of the slab. the displacements of the material points initially change rapidly with time. However. the displacements change less rapidly as time goes on. eventually reaching an asymptotic value. We find that the inhomogeneous deformations obtained by Rajagopal and Wineman (1985) within the context of Neo-Hookean and Mooney-Rivlin theories of elasticity can be recovered as special cases of the solutions established here.

In Section 2, the constitutive equation for the non-linear viscoelastic material to be employed is introduced and the non-uniform extension problems corresponding to the relaxation and creep problems are formulated. The problems are reduced to solving nonlinear fourth order partial differential equations. The details of the numerical method are given in Section 3 and the results are discussed in Section 4 .

\section{FORMULATION}

We shall assume that the Cauchy stress $\sigma$ has the form [cf. Pipkin and Rogers (1968)]

$$
\sigma=-p \mathbf{I}+\mathbf{F}(t)\left\{\mathbf{R}[\mathbf{C}(t) ; 0]+\int_{0}^{t} \frac{\partial}{r(t-s)} \mathbf{R}[\mathbf{C}(s), t-s] \mathrm{d} s\right\} \mathbf{F}^{\mathrm{T}}(t)
$$

where the term $-p$ is due to the constraint of incompressibility, $F$ is the deformation gradient tensor and $\mathbf{C}=\mathbf{F} \mathrm{r}, \mathbf{K}[\mathbf{C}, \ell]$ is the strain dependent tensorial relaxation function induced by a single step strain history and has the form

$$
\mathbf{R}=\phi_{0} \mathbf{I}+\phi_{1} \mathbf{C}+\phi_{2} \mathbf{C}^{2}
$$

where $\phi_{n}, \phi_{1}, \phi_{2}$ are scalar functions of $t$ and the invariants of $\mathrm{C}$. Pipkin and Rogers (1968) did not, however, present any specific forms for the strain dependent relaxation functions $\phi_{t,} i=0,1,2$, appearing in (2). Wineman (1972) chose $\phi_{i}$ such that

$$
\mathbf{R}[\mathbf{C}(s), \check{\zeta}]=R(\xi)\left\{\left[1+\mu l(s) \mid \mathbf{1}-\mu \mathrm{C}(s)_{j}^{\prime},\right.\right.
$$

where

$$
l(s)=\operatorname{tr} C(s), \quad R(\xi)=C_{0}\left[(1-\gamma) \exp \left(-\frac{\xi}{\tau_{R}}\right)+\gamma\right], \quad \mu>0,0<\gamma<1,
$$

where $R\left({ }_{\zeta}\right)$ is a relaxation function associated with small strains, and $y=C_{x} / C_{11}, C_{0}$ denotes the initial modulus and $C_{2}$ the residual modulus. Note that if time dependence is suppressed from (1) with (3) and (4), it reduces to a Mooney-Rivlin material, in which $\mu$ represents the derivative of the strain energy density function with respect to the second strain invariant divided by the derivative with respect to the first strain invariant. As will be seen, this feature enables a discussion of non-homogeneous deformations in the present context, to be related to the discussion of non-homogeneous deformations for the elastic problem. Equation (1) with (3) and (4) can be rewritten in the form

$$
\begin{aligned}
\sigma=-p \mathbf{I}+C_{n}\left\{[1+\mu J(t)] \mathbf{B}(t)-\mu \mathbf{B}^{2}(t)\right\}-\frac{C_{0}(1-\gamma)}{\tau_{\mathrm{R}}} & \int_{0}^{t} \exp \left(-\begin{array}{c}
t-s \\
\tau_{\mathrm{R}}
\end{array}\right) \\
& \times\left\{[1+\mu I(s)] \mathbf{B}(t)-\mu \mathbf{F}(t) \mathbf{C}(s) \mathbf{F}^{\mathrm{T}}(t)\right\} \mathrm{d} s .
\end{aligned}
$$

Consider a viscoelastic slab which undergoes the following deformation :

$$
x=\frac{X}{\sqrt{\lambda^{\prime}(Z, t)}}, \quad y=\frac{Y}{\sqrt{\lambda^{\prime}(Z . t)}}, \quad z=\lambda(Z, t),
$$


where prime denotes the partial derivative with respect to $Z$. This deformation allows for non-homogeneous deformation along the $Z$-axis and uniform contraction or expansion on surfaces $Z=$ constant. The deformation gradient $F$ is given by

$$
\mathbf{F}=\left[\begin{array}{ccc}
\frac{1}{\sqrt{\lambda^{\prime}}} & 0 & \alpha \\
0 & \frac{1}{\sqrt{\lambda^{\prime}}} & \beta \\
0 & 0 & \lambda^{\prime}
\end{array}\right]
$$

where

$$
\begin{aligned}
& x=-\frac{1}{2}\left(\lambda^{\prime}\right)^{-3 / 2} \lambda^{\prime \prime} X . \\
& \beta=-\frac{1}{2}\left(\lambda^{\prime}\right)^{-3 / 2} \lambda^{\prime \prime} Y,
\end{aligned}
$$

and thus $\mathbf{F}$ depends on $X, Y$ and $Z$. The Left Cauchy-Green deformation tensor $\mathbf{B}=\mathbf{F F}^{\top}$ has the following matrix representation:

$$
\mathbf{B}=\mathbf{F F}=\left[\begin{array}{ccc}
\frac{1}{\lambda^{\prime}}+\alpha^{2} & \alpha \beta & \alpha \lambda^{\prime} \\
-\alpha \beta & \frac{1}{\lambda^{\prime}}+\beta^{2} & \beta \lambda^{\prime} \\
\alpha \lambda^{\prime} & \beta \lambda^{\prime} & \lambda^{\prime 2}
\end{array}\right]
$$

Thus,

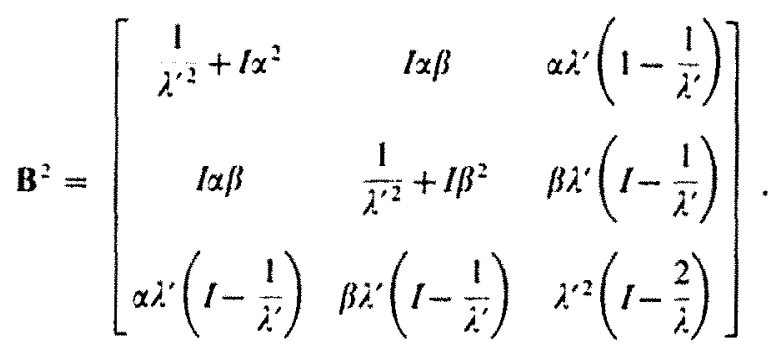

where

$$
I \equiv \operatorname{tr} B \equiv \frac{2}{\lambda^{\prime}}+\alpha^{2}+\beta^{2}+\left(\lambda^{\prime}\right)^{2}
$$

The Right Cauchy-Green deformation tensor $\mathbf{C}=\mathrm{F}^{\mathrm{T}} \mathbf{F}$ is given by

$$
\mathbf{C}=\left[\begin{array}{ccc}
\frac{1}{\lambda^{\prime}} & 0 & \frac{\alpha}{\sqrt{\lambda^{\prime}}} \\
0 & \frac{1}{\lambda^{\prime}} & \frac{\beta}{\sqrt{\lambda^{\prime}}} \\
\frac{\alpha}{\sqrt{\lambda^{\prime}}} & \frac{\beta}{\sqrt{\lambda^{\prime}}} & I-\frac{2}{\lambda^{\prime}}
\end{array}\right] .
$$


Also, the tensor $\mathbf{F}^{-1}$ has the matrix representation:

$$
\mathbf{F}^{-1}=\left[\begin{array}{ccc}
\sqrt{\lambda} & 0 & -\frac{x}{\lambda} \\
0 & \sqrt{\lambda} & -\frac{\beta}{\sqrt{\lambda}} \\
0 & 0 & \frac{1}{\eta}
\end{array}\right] .
$$

For notational convenience, let $\mathbf{M}=\mathbf{F}(t) \mathbf{C}(s) \mathbf{F}^{\mathrm{T}}(t)$. From (7) and (13). we observe that the components of $\mathbf{M}$ are:

$$
\begin{aligned}
& M_{11}=x^{2}\left[I(s)-\frac{2}{\lambda^{\prime}(s)}\right]+\frac{1}{\lambda^{\prime}(s) \lambda^{\prime}}+\frac{2 x(s) x}{\sqrt{\lambda^{\prime}(s) \lambda^{\prime}}} \\
& M_{12}=M_{21}=\alpha \beta\left[I(s)-\frac{2}{\lambda^{\prime}(s)}\right]+\frac{\chi(s) \beta}{\sqrt{\lambda^{\prime}(s) \lambda^{\prime}}}+\frac{\beta(s) \alpha}{\sqrt{\lambda^{\prime}(s) \lambda^{\prime}}} \\
& M_{13}=M_{11}=x i \cdot\left[I(s)-\frac{2}{\lambda^{\prime}(s)}\right]+\frac{\lambda^{\prime} x(s)}{\sqrt{\lambda(s) \dot{\lambda}}} \\
& M_{22}=\beta^{2}\left[l(s)-\begin{array}{c}
2 \\
\lambda^{\prime}(s)
\end{array}\right]+\frac{1}{\lambda^{\prime}(s) \lambda^{\prime}}+\frac{2 \beta(s) \beta}{\lambda^{\prime}(s) \lambda^{\prime}}
\end{aligned}
$$

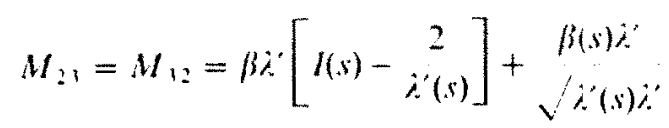

$$
\begin{aligned}
& M_{3}=\left(\lambda^{\prime}\right)^{2}\left[I(s)-z^{\prime}(s)\right]
\end{aligned}
$$

where

$$
\begin{gathered}
I(s)=\frac{2}{\lambda^{\prime}(s)}+x^{2}(s)+\beta^{2}(s)+\left[\lambda^{\prime}(s)\right]^{2}, \quad x(s)=-\frac{1}{2}\left[\lambda^{\prime}(s)\right]^{-3} \lambda^{\prime \prime}(s) X, \\
\beta(s)=-\frac{1}{2}\left[\lambda^{\prime}(s)\right] \lambda^{\prime \prime} \lambda^{\prime \prime}(s) Y, \quad \lambda^{\prime}(s)=\frac{\partial \lambda(Z, s)}{\partial Z}
\end{gathered}
$$

We shall assume that inertial effects in the equations of motion can be neglected as quantities vary slowly with time. In view of the assumed form of the deformation, we find that it is computationally more convenient to express the equations of motion in terms of the reference configuration. Then, in the absence of body forces, the equations of motion take the form

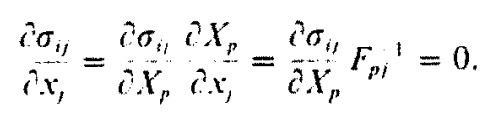

From (5) and (7)-(17), a lengthy but straightforward calculation yields the following equations:

$$
\begin{aligned}
& \frac{\partial p}{\partial X}=X f(Z, t) . \\
& \frac{\partial p}{\partial Y}=Y f(Z, t) .
\end{aligned}
$$




$$
\begin{aligned}
\frac{\partial p}{\partial Z}=-\frac{1}{2} \frac{\lambda^{\prime \prime}}{\lambda^{\prime}} & \left(X^{2}+Y^{2}\right) f(Z, t)-C_{0}\left(1+\frac{\mu}{\dot{\lambda}^{\prime}}\right) \lambda^{\prime} \lambda^{\prime \prime} \\
& +\frac{C_{0}(1-\eta)}{\tau_{\mathrm{R}}} \int_{0}^{t} \exp \left(-\frac{t-s}{\tau_{\mathrm{R}}}\right)\left\{\left[1+\frac{2 \mu}{\lambda^{\prime}(s)}\right] \frac{\lambda^{\prime \prime}}{\lambda^{\prime}}-\mu \frac{\lambda^{\prime \prime}(s)}{\left[\lambda^{\prime}(s)\right]^{2}}\right\}\left(\lambda^{\prime}\right)^{2} \mathrm{~d} s \\
& +C_{0} \frac{\partial}{\partial Z}\left\{\left(1+\frac{2 \mu}{\lambda^{\prime}}\right)\left(\lambda^{\prime}\right)^{2}-\frac{(1-\gamma)}{\tau_{\mathrm{R}}} \int_{0}^{t} \exp \left(-\frac{t-s}{\tau_{\mathrm{R}}}\right)\left[1+\frac{2 \mu}{\lambda^{\prime}(s)}\right]\left(\lambda^{\prime}\right)^{2} \mathrm{~d} s\right\},
\end{aligned}
$$

where

$$
\begin{aligned}
f(Z, t)= & C_{0}\left(2+\frac{\mu}{\lambda^{\prime}}\right)\left[-\frac{1}{2}\left(\lambda^{\prime}\right)^{-3 / 2} \lambda^{\prime \prime}\right]^{2}-\frac{C_{0}(1-\eta)}{\tau_{\mathrm{R}}} \int_{0}^{t} \exp \left(-\frac{t-s}{\tau_{\mathrm{R}}}\right) \\
& \times\left\{\left[2+\frac{4 \mu}{\lambda^{\prime}(s)}\right]\left[-\frac{1}{2}\left(\lambda^{\prime}\right)^{-3 / 2} \lambda^{\prime \prime}\right]^{2}-\frac{5 \mu}{\sqrt{\lambda^{\prime}(s) \lambda^{\prime}}}\left\{-\frac{1}{2}\left[\lambda^{\prime}(s)\right]^{-3 / 2} \lambda^{\prime \prime}(s)\right\}\left[-\frac{1}{2}\left(\lambda^{\prime}\right)^{-3 / 2} \lambda^{\prime \prime}\right]\right. \\
& \left.+\frac{2 \mu}{\lambda^{\prime}}\left\{-\frac{1}{2}\left[\lambda^{\prime}(s)\right]^{-3 / 2} \lambda^{\prime \prime}(s)\right\}^{2}\right\} \mathrm{d} s+\frac{C_{0}}{\left(\lambda^{\prime}\right)^{3 / 2}} \frac{\partial}{\partial Z}\left\{\left(\lambda^{\prime}+\mu\right)\left[-\frac{1}{2}\left(\lambda^{\prime}\right)^{-3 / 2} \lambda^{\prime \prime}\right]\right. \\
& -\frac{C_{0}(1-\gamma)}{\tau_{\mathrm{R}}} \int_{0}^{t} \exp \left(-\frac{1-s}{\tau_{\mathrm{R}}}\right)\left\{\lambda^{\prime}\left[1+\frac{2 \mu^{\prime}}{\lambda^{\prime}(s)}\right]\left[-\frac{1}{2}\left(\lambda^{\prime}\right)^{-3 / 2} \lambda^{\prime \prime}\right]\right. \\
& \left.\left.-\mu \sqrt{\frac{\lambda^{\prime}}{\lambda^{\prime}(s)}}\left\{-\frac{1}{2}\left[\lambda^{\prime}(s)\right]^{-3 / 2} \lambda^{\prime \prime}(s)\right\}\right\} \mathrm{d} s\right\}=0 .
\end{aligned}
$$

The scalar $p$ can be eliminated from (18) and (20) to obtain

$$
\frac{\partial f(Z, t)}{\partial Z}=-\frac{\lambda^{\prime \prime}}{\lambda^{\prime}} f(Z, t)
$$

This same result can also be obtained from (19) and (20). Integration of (22) yields

$$
\lambda^{\prime} f=C_{1}(t)
$$

where $C_{1}(t)$ is an arbitrary function of $t$. It follows from (21) and (23) that

$$
\begin{aligned}
& \mu \frac{\left(\lambda^{\prime \prime}\right)^{2}}{\left(\lambda^{\prime}\right)^{3}}+\frac{3}{4}\left(\frac{\lambda^{\prime \prime}}{\lambda^{\prime}}\right)^{2}-\frac{1}{2}\left(\lambda^{\prime}+\mu\right) \frac{\lambda^{\prime \prime \prime}}{\left(\lambda^{\prime}\right)^{2}}-\frac{(1-\gamma)}{\tau_{\mathrm{R}}} \int_{0}^{t} \exp \left(-\frac{t-s}{\tau_{\mathrm{R}}}\right) \\
& \quad \times\left\{-\frac{\mu}{2} \frac{\left[\lambda^{\prime \prime}(s)\right]^{2}}{\left[\lambda^{\prime}(s)\right]^{3}}+\frac{3}{4}\left[1+\frac{2 \mu}{\lambda^{\prime}(s)}\right]\left(\frac{\lambda^{\prime \prime}}{\lambda^{\prime}}\right)^{2}-\frac{1}{2}\left[1+\frac{2 \mu}{\lambda^{\prime}(s)}\right] \frac{\lambda^{\prime \prime \prime}}{\lambda^{\prime}}+\frac{\mu}{2} \frac{\lambda^{\prime \prime \prime}(s)}{\left[\lambda^{\prime}(s)\right]^{2}}\right\} \mathrm{d} s=C(t),
\end{aligned}
$$

where $C(t)=C_{1}(t) / C_{0}$.

Equation (24) is a Volterra-integro differential equation for $\lambda(Z, t)$ which is third order in the spatial derivative. It also contains the arbitrary function $C(t)$. This implies that four boundary conditions are needed. Suppose we consider the case of a viscoelastic slab which is originally bounded by the planes $Z=H$ and $Z=0$. Appropriate conditions at the bottom and top boundaries, respectively, would be

$$
\lambda(0, t)=0, \lambda(H, t)=h(t)
$$

and

$$
\lambda^{\prime}(0, t)=g(t), \quad \lambda^{\prime}(H, t)=f(t) .
$$


Condition (25), states that the slab initially at $Z=0$ cannot move in the $Z$-direction. Condition (25): states that the slab initially at $Z=H$ moves to $z=h$ at time $t$. From (6), it can be seen that the boundary conditions $(26)_{1}$ and $(26)_{2}$ are equivalent to prescribing the uniform contraction or expansion of the plane surfaces at the bottom and top. respectively. It is also possible to specify normal tractions $\sigma_{z:}(0,0,0, t), \sigma_{z:}(0,0, H, t)$ as alternate boundary conditions. They would then replace the appropriate conditions of (25) or (26).

Let us turn our attention to determining the unknown scalar $p$, and then the stresses. From (18)-(20) and (23). we have

$$
\begin{aligned}
p(X . Y . Z . t) & =\frac{1}{2}\left(X^{2}+Y^{2}\right) f(Z, t)+b(Z, t) \\
& =\frac{1}{2}\left(X^{2}+Y^{2}\right) \frac{C_{1}(t)}{\lambda^{\prime}}+b(Z, t),
\end{aligned}
$$

where

$$
\begin{aligned}
b(Z, t)=C_{0}\left\{\left(\frac{1}{2} \lambda^{\prime}+\mu\right) \lambda^{\prime}-!(1-\gamma)\left(\lambda^{\prime}\right)^{2}\right. & {\left[1-\exp \left(-\frac{t}{\tau_{R}}\right)\right] } \\
& \left.-\frac{\left(1-\gamma^{\prime}\right) \mu}{\tau_{R}} \int_{0}^{t} \exp \left(-\frac{t-s}{\tau_{R}}\right) \frac{\left(\lambda^{\prime}\right)^{2}}{\lambda^{\prime}(s)} \mathrm{d} s\right\}+C_{2}(t) .
\end{aligned}
$$

and $C_{2}(t)$ is an arbitrary function obtained duc to integration. Substituting (10), (11), (15) and (27) with (28) into (5), we have

$$
\begin{aligned}
& \sigma_{1}(X, Y, Z, t) / C_{0}=\sigma_{w}(X, Y, Z, t) / C_{0}=C_{2}(t)-\frac{1}{2}\left(\lambda^{\prime}\right)^{2}+\frac{1}{2}(1-\gamma)\left[\left(\lambda^{\prime}\right)^{2}-\frac{2}{\lambda^{\prime}}-\frac{\lambda^{\prime \prime}}{2\left(\lambda^{\prime}\right)^{\prime}} X^{\prime 2}\right] \\
& \times\left[1-\exp \left(-\frac{1}{t_{\mathrm{R}}}\right)\right]+\frac{1}{\lambda^{\prime}}\left(1+\frac{1}{\lambda^{\prime}}\right)+\frac{\lambda^{\prime \prime}}{4\left(\lambda^{\prime}\right)^{3}} x^{2} \\
& +\frac{1}{2 \lambda^{\prime}}\left[\frac{\mu\left(\lambda^{\prime \prime}\right)^{2}}{2\left(\lambda^{\prime}\right)^{3}}-C_{1}(t)\right]\left(X^{2}+Y^{2}\right)+\frac{(1-\gamma) \mu}{\tau_{\mathrm{R}}} \int_{0}^{t} \exp \left(-\frac{t-s}{\tau_{\mathrm{R}}}\right) \\
& \times\left\{\frac{\left(\lambda^{\prime}\right)^{2}}{\lambda^{\prime}(s)}-\frac{1}{\lambda^{\prime}(s) \lambda^{\prime}}-\frac{\left[\lambda^{\prime}(s)\right]^{2}}{\lambda^{\prime}}+\frac{\lambda^{\prime \prime}}{2 \lambda^{\prime}(s)\left(\lambda^{\prime}\right)^{2}}\left[\frac{\lambda^{\prime \prime}(s)}{\lambda^{\prime}(s)}-\frac{\lambda^{\prime \prime}}{\lambda^{\prime}}\right] X^{2}-\frac{1}{4} \frac{\left[\lambda^{\prime \prime}(s)\right]^{2}}{\lambda^{\prime}\left[\lambda^{\prime}(s)\right]^{3}}\left(X^{2}+Y^{\prime}\right)\right\} \mathrm{d} s,
\end{aligned}
$$

$$
\begin{aligned}
\sigma_{1:}(X, Y, Z, t) / C_{0}=- & \frac{1}{2}\left\{\lambda^{\prime}+\mu-C_{0}(1-\gamma)\left[1-\exp \left(-\frac{t}{\tau_{\mathrm{R}}}\right)\right] \lambda^{\prime}\right\} \frac{\lambda^{\prime \prime} X}{\sqrt{\lambda^{\prime 3}}} \\
& +\frac{C_{0}\left(1-\gamma^{\prime}\right) \mu}{\tau_{\mathrm{R}}} \frac{\lambda^{\prime \prime} X}{\sqrt{\lambda^{\prime}}} \int_{0}^{t} \frac{1}{\lambda^{\prime}(s)}\left[1-\frac{1}{2} \frac{\lambda^{\prime \prime}(s) \lambda^{\prime}}{\lambda^{\prime}(s) \lambda^{\prime \prime}}\right] \exp \left(-\frac{t-s}{\tau_{\mathrm{R}}}\right) \mathrm{d} . s
\end{aligned}
$$

and

$$
\begin{aligned}
\sigma_{::}(X, Y, Z, t) / C_{0}=C_{2}(t)-\frac{C_{1}(t)}{2 \lambda^{\prime}}\left(X^{2}+Y^{2}\right)+\left(\frac{1}{2} \lambda^{\prime}+\mu\right) \lambda^{\prime}-\frac{1}{2}(1-\gamma)\left(\lambda^{\prime}\right)^{2}\left[1-\exp \left(-\frac{t}{\tau_{\mathrm{R}}}\right)\right] \\
-\frac{(1-\gamma) \mu}{\tau_{\mathrm{R}}} \int_{0}^{\prime} \frac{\left(\lambda^{\prime}\right)^{2}}{\lambda^{\prime}(s)} \exp \left(-\frac{t-s}{\tau_{\mathrm{R}}}\right) \mathrm{d} s
\end{aligned}
$$

Equation (24) admits the solution corresponding to a homogeneous uniaxial extension history, $\lambda^{\prime}(Z . t)=\lambda_{0}^{\prime}(t)$. in which case $C(t)=0$. Conversely, if $C(t)=0$. the uniaxial 
extension history need not be homogeneous. In order to show this. let $t=0$ in eqn (24). This reduces to the same equation as for the elastic problem for a Mooney-Rivlin material [cf. Rajagopal et al. (1986)]. When $\mu=0$, these equations reduce further to the case of a Neo-Hookean material [cf. Rajagopal and Wineman (1985)]. The results in this paper imply that, if $C(0)=0$, then

$$
\lambda^{\prime}(Z)=\frac{1}{\left(x_{1} Z+x_{2}\right)^{2}} .
$$

where $\alpha_{1}$ and $\alpha_{2}$ are constants. Thus in the case of a Neo-Hookean material, when $C=0$. the stretch ratio $\lambda^{\prime}$ need not be uniform. The classical solution $\lambda^{\prime}=$ constant is a special subclass which corresponds to $\alpha_{1}=0$. Similarly, non-uniform solutions for the stretch ratio are possible in a Mooney-Rivlin material, the classical uniform solution being a special subclass of the same.

Suppose that there are no stresses in the viscoelastic slab. if there is null deformation history. Mathematically, this implies that $\sigma_{i j}=0$. if $\lambda^{\prime}=\lambda^{\prime}(s)=1$ and $\lambda^{\prime \prime}=\lambda^{\prime \prime}(s)=0$. When $\lambda^{\prime \prime}=\lambda^{\prime \prime}(s)=0$, by virtue of $(24), C(t)=0$. Then it follows from (29) or (3l) that

$$
C_{2}(t)=-\left(\frac{1}{2}+\mu\right)\left\{1-(1-\gamma)\left[1-\exp \left(-\frac{t}{t_{R}}\right)\right]\right\} \text {. }
$$

\section{NUMERICAL METHOD}

The method developed by Lee and Rogers (1963) to solve boundary problems of linear viscoelasticity was successfully extended to the solution of non-linear viscoclastic problems by Wineman (1972). Here, this method is further improved [cf. Ferziger (1981)]. For convenience in developing this method, we express (24) in the form

$$
\begin{aligned}
\frac{\partial \lambda^{\prime \prime}}{\partial Z}\left\{F_{1}\left(\lambda^{\prime}\right)+\int_{0}^{t} G_{1}\left[\lambda^{\prime}(s) ; t-s\right] \mathrm{d} s\right\} & +\int_{0}^{t} \frac{\partial \lambda^{\prime \prime}(s)}{\partial Z} G_{2}\left[\lambda^{\prime} ; \lambda^{\prime}(s) ; t-s\right] \mathrm{d} s \\
& +F_{2}\left(\lambda^{\prime}, \lambda^{\prime \prime}\right)+\int_{0}^{t} G_{3}\left[\lambda^{\prime} ; \lambda^{\prime \prime} ; \lambda^{\prime}(s) ; \lambda^{\prime \prime}(s) ; t-s\right] \mathrm{d} s=0,
\end{aligned}
$$

where

$$
\begin{aligned}
& F_{1}=\frac{1}{2}\left(1+\frac{\mu}{\lambda^{\prime}}\right), \\
& G_{1}=-\frac{1}{2} \frac{(1-\gamma)}{\tau_{\mathrm{R}}}\left[1+\frac{2 \mu}{\lambda^{\prime}(s)}\right] \exp \left(-\frac{t-s}{\tau_{\mathrm{R}}}\right), \\
& G_{2}=\frac{\mu(1-\gamma)}{2 \tau_{\mathrm{R}}} \frac{\lambda^{\prime}}{\left[\lambda^{\prime}(s)\right]^{2}} \exp \left(-\frac{t-s}{\tau_{\mathrm{R}}}\right), \\
& F_{2}=-\mu\left(\frac{\lambda^{\prime \prime}}{\lambda^{\prime}}\right)^{2}-\frac{3}{4} \frac{\left(\dot{\lambda}^{\prime \prime}\right)^{2}}{\lambda^{\prime}}+C(t) \dot{\lambda}^{\prime}, \\
& G_{3}=-\left\{\frac{\mu}{2} \frac{\left[\lambda^{\prime \prime}(s)\right]^{2} \lambda^{\prime}}{\left[\lambda^{\prime}(s)\right]^{3}}-\frac{3}{4}\left[1+\frac{2 \mu}{\lambda^{\prime}(s)}\right] \frac{\left(\lambda^{\prime \prime}\right)^{2}}{\lambda^{\prime}}\right\} \frac{(1-\gamma)}{\tau_{\mathrm{R}}} \exp \left(-\frac{t-s}{\tau_{\mathrm{R}}}\right) .
\end{aligned}
$$

Let the interval $[0, t]$ be partitioned into $n$ subintervals $\left[t_{1}=0, t_{2}, \ldots, t_{n}=t\right]$. The third 
integral in (34) can be written as

$$
\int_{0}^{t} G_{3}\left[\lambda^{\prime}: \lambda^{\prime \prime} ; \lambda^{\prime}(s) ; \lambda^{\prime \prime}(s) ; t-s\right] \mathrm{d} s=\int_{t_{1}}^{t_{n}} G_{3}\left[\lambda^{\prime}\left(t_{n}\right) ; \lambda^{\prime \prime}\left(t_{n}\right) ; \lambda^{\prime}(s) ; \lambda^{\prime \prime}(s) ; t_{n}-s\right] \mathrm{d} s
$$

Expressing (36) as a summation of $n-1$ integrals over the subintervals $\left(t_{k}, t_{k+1}\right)$. $(k=1, n-1)$, approximating each of these by the trapezoidal rule and denoting the finite sum approximation to the third integral of (34) by $S_{i, \text { we have }}$

$$
\begin{aligned}
S_{3}= & \int_{0}^{t} G_{3}\left[\lambda^{\prime} ; \lambda^{\prime \prime} ; \lambda^{\prime}(s) ; \lambda^{\prime \prime}(s) ; t-s\right] \mathrm{d} s \\
\approx & \frac{1}{2}\left\{G_{3}\left[\lambda^{\prime}\left(t_{n}\right), \lambda^{\prime \prime}\left(t_{n}\right), \lambda^{\prime}\left(t_{n}\right), \lambda^{\prime \prime}\left(t_{n}\right), 0\right]\left(t_{n}-t_{n-1}\right)\right. \\
& +G_{3}\left[\lambda^{\prime}\left(t_{n}\right), \lambda^{\prime \prime}\left(t_{n}\right), \lambda^{\prime}\left(t_{1}\right), \lambda^{\prime \prime}\left(t_{1}\right), t_{n}-t_{1}\right]\left(t_{2}-t_{1}\right) \\
& \left.+\sum_{k=2}^{n-1} G_{3}\left[\lambda^{\prime}\left(t_{n}\right), \lambda^{\prime \prime}\left(t_{n}\right), \lambda^{\prime}\left(t_{k}\right), \lambda^{\prime \prime}\left(t_{k}\right), t_{n}-t_{k}\right]\left(t_{k+1}-t_{k-1}\right)\right\} .
\end{aligned}
$$

Similarly, the first two integrals of (34) can also be written in the same form as (36). For notational convenience, we denote the finite sum approximation to the first integral of (34) by $S_{1}$. Letting $S_{2}$ denote only the terms in the approximation to the second integral which contain $\lambda\left(t_{k}\right), k<n$, we have

$$
\begin{aligned}
S_{1}= & \int_{0}^{t} G_{1}\left[\lambda^{\prime}(s) ; t-s\right] \mathrm{d} s \\
\approx & \sum_{2}\left\{G_{1}\left[\lambda^{\prime}\left(t_{n}\right), 0\right]\left(t_{n}-t_{n-1}\right)+G_{1}\left[\lambda^{\prime}\left(t_{1}\right), t_{n}-t_{1}\right]\left(t_{2}-t_{1}\right)\right. \\
& \left.+\sum_{k=2}^{n-1} G_{1}\left[\lambda^{\prime}\left(t_{k}\right), t_{n}-t_{k}\right]\left(t_{k+1}-t_{k} 1\right)\right\}
\end{aligned}
$$

and

$$
\int_{0}^{t} \frac{\partial \lambda^{\prime \prime}(s)}{\partial Z} G_{2}\left[\lambda^{\prime} ; \lambda^{\prime}(s) ; t-s\right] \mathrm{d} s \approx \frac{\partial \lambda^{\prime \prime}\left(t_{n}\right)}{\partial Z} \hat{G}_{2}\left[\lambda^{\prime}\left(t_{n}\right), \lambda^{\prime}\left(t_{n}\right), 0\right]+S_{2}
$$

where

$$
\begin{gathered}
\hat{G}_{2}\left[\lambda^{\prime}\left(t_{n}\right), \lambda^{\prime}\left(t_{n}\right), 0\right]=\frac{1}{2} G_{2}\left[\lambda^{\prime}\left(t_{n}\right) ; \lambda^{\prime}\left(t_{n}\right) ; 0\right]\left(t_{n}-t_{n}\right), \\
S_{2}=1\left\{G_{2}\left[\lambda^{\prime}\left(t_{n}\right), \lambda^{\prime}\left(t_{1}\right), t_{n}-t_{1}\right]\left(t_{2}-t_{1}\right) \frac{\partial \lambda^{\prime \prime}\left(t_{1}\right)}{\partial Z}\right. \\
\left.+\sum_{k=2}^{n} G_{2}\left[\lambda^{\prime}\left(t_{n}\right), \lambda^{\prime}\left(t_{k}\right), t_{n}-t_{k}\right]\left(t_{k+1}-t_{k-1}\right) \frac{\partial^{\prime \prime}\left(t_{k}\right)}{\partial Z}\right\} .
\end{gathered}
$$

In the finite sum approximation to the second integral of (34), it can be seen from (41) that the term depending on $\lambda^{\prime}\left(t_{k}\right)$ has $\partial \lambda^{\prime \prime}\left(Z, t_{k}\right) / \partial Z$ as a coefficient. For $t_{k}<t_{n}$. this derivative is approximated by a simple difference expression. 
The method of solution will be to determine $\vec{c} \dot{\lambda}^{\prime \prime}(Z . t)_{i}^{\prime} \bar{C} Z$ at each time $t_{n}$ in terms of $\lambda^{\prime}(Z, t)$ and $\lambda^{\prime \prime}(Z, t)$ and the solutions determined at previous time steps $t_{k}<t_{n}$. We first consider the method of solution for the initial response at $t_{n}=t_{1}=0$. Equation (34) can be reduced to the following system of first order ordinary differential equations. From (34). the initial elastic response, $t_{n}=t_{1}=0$, satisfies the following system of equations:

$$
\frac{\partial \lambda\left(t_{1}\right)}{\partial Z}=\lambda_{1}\left(t_{1}\right) . \quad \frac{\partial \lambda_{1}\left(t_{1}\right)}{\partial Z}=\lambda_{2}\left(t_{1}\right) . \quad \frac{\partial \lambda_{2}\left(t_{1}\right)}{\partial Z}=-\frac{F_{2}\left[\lambda_{1}\left(t_{1}\right), \lambda_{2}\left(t_{1}\right)\right]}{F_{1}\left[\lambda_{1}\left(t_{1}\right)\right]} .
$$

This system can be integrated by fourth order Runge-Kutta method [cf. William (1986)] subject to conditions (25) and (26). Starting values for the numerical integration. given by $(25)_{1}$ and $(26)_{1}$, are $\lambda\left(0, t_{1}\right)=0$ and $\lambda_{1}\left(0, t_{1}\right)=\lambda^{\prime}\left(0, t_{1}\right)=g\left(t_{1}\right)$. The starting value $\lambda_{2}\left(0, t_{1}\right)=\lambda^{\prime \prime}\left(0, t_{1}\right)$ and $C\left(t_{1}\right)$ can then be determined so as to satisfy the end conditions required by $(25)_{2}$ and $(26)_{2}$. namely $\lambda\left(H, t_{1}\right)=h\left(t_{1}\right)$ and $\lambda_{1}\left(H, t_{1}\right)=\lambda^{\prime}\left(H, t_{1}\right)=f\left(t_{1}\right)$. This requires a two-dimensional shooting method. In applying this method, a value is assumed for $C\left(t_{1}\right)$ and then $\lambda_{2}\left(0, t_{1}\right)$ is adjusted so as to satisfy $(25)_{2}$. The process is repeated with new values of $C\left(t_{1}\right)$ until $(26)_{2}$ is satisfied. However, for the purpose of this work, a value of $C\left(t_{1}\right)$ is assumed, and the value of $f\left(t_{1}\right)$ is found by use of $(26)_{2}$.

Note that normal tractions $\sigma_{: z}\left(0,0,0, t_{1}\right)$ and $/$ or $\sigma_{::}\left(0,0, H, t_{1}\right)$ could be specified in place of (26). By (31), these conditions could be solved for the values of $\lambda_{1}\left(0, t_{1}\right)$ and/or $\lambda_{1}\left(H, t_{1}\right)$. The traction conditions can thus be considered equivalent to (26). If $\left|\lambda\left(H, t_{1}\right)-h\left(t_{1}\right)\right|=\delta>\delta$, for some prescribed $\delta . \lambda^{\prime \prime}\left(0, t_{1}\right)$ is adjusted and the process is repeated. $\lambda "\left(0, t_{1}\right)$ is chosen and automatically adjusted as follows. Let an initial guess of $\lambda^{\prime \prime}\left(0, t_{1}\right)$ be denoted by $h$. Then $\lambda^{\prime \prime}\left(0, t_{1}\right)$ is assigned the following three values $-h$, $2 b \times 0.618-b$ and $b$, in turn. Suppose that $2 h \times 0.618-b$ and $h$ are the two values which result in the two smallest values or the crror $\delta$. Values 0 and $b$ are picked up as the end points of the new range of $\lambda^{\prime \prime}\left(0 . l_{1}\right)$ in the second iterattion. Then. $0, b \times 0.618$ and $b$ are assigned to $\lambda^{\prime \prime}\left(0, t_{1}\right)$ ats its new values in turn. Now if $h \times 0.618$ and $h$ are the most recent values which result in the new smallest values of $\delta, b / 2$ and $b$ are chosen als the end points of the range of $\lambda^{\prime \prime}\left(0, l_{1}\right)$ for the third itcration. This time. the values $b / 2, b / 2(1.0+0.618)$ and $h$ are assigned to $\lambda^{\prime \prime}\left(0, l_{1}\right)$, in turn. This procedure is continued until al value of $\lambda^{\prime \prime}\left(0, t_{1}\right)$ is found which results in $\left|\lambda\left(H, t_{1}\right)-h\left(t_{1}\right)\right|=\delta<\varepsilon$. It should be pointed out that $h$ should be chosen large enough so that $[-b, b]$ will cover a large enough range of values for $\lambda^{\prime \prime}\left(0, t_{1}\right)$.

For $n>2$, eqn (34) is written in terms of the notations defined in (37)-(41) as

$$
\frac{\partial \lambda^{\prime \prime}\left(t_{n}\right)}{\partial Z}\left\{F_{1}\left[\lambda^{\prime}\left(t_{n}\right)\right]+S_{1}\right\}+\frac{\partial \lambda^{\prime \prime}\left(t_{n}\right)}{\partial Z} \hat{G}_{2}\left[\lambda^{\prime}\left(t_{n}\right), \lambda^{\prime}\left(t_{n}\right), 0\right]+S_{2}+F_{2}\left[\lambda^{\prime}\left(t_{n}\right) \cdot \lambda^{\prime \prime}\left(t_{n}\right)\right]+S_{3}=0 .
$$

Furthermore, eqn (43) is rewritten in the form:

$$
\frac{\partial \lambda_{(}\left(t_{n}\right)}{\partial Z}=\lambda_{1}\left(t_{n}\right), \quad \frac{\partial \lambda_{1}\left(t_{n}\right)}{\partial Z}=\lambda_{2}\left(t_{n}\right), \quad \frac{\partial \lambda_{2}\left(t_{n}\right)}{\partial Z}=-\frac{F_{2}\left[\lambda_{1}\left(t_{n}\right) \lambda_{2}\left(t_{n}\right)\right]+S_{3}+S_{2}}{F_{1}\left[\lambda_{1}\left(t_{n}\right)\right]+S_{1}+\hat{G}_{2}\left[\lambda_{1}\left(t_{n}\right), \lambda_{1}\left(t_{n}\right), 0\right]} .
$$

In the third of equations (44), $F_{1}, F_{2}$ and $\hat{G}_{2}$ depend only on $i^{\prime}\left(t_{n}\right)$ and $\lambda^{\prime \prime}\left(t_{n}\right)$, while $S_{1}$, $S_{2}$ and $S_{3}$ depend on $\lambda^{\prime}\left(t_{n}\right) . \lambda^{\prime \prime}\left(t_{n}\right) . \lambda^{\prime}\left(t_{k}\right) . \lambda^{\prime \prime}\left(t_{k}\right)$ and $\partial \lambda^{\prime \prime}\left(t_{k}\right) / \partial Z, k<n$. Because the last three have been found by solving (44) for times $t_{k}<t_{n}, S_{1}, S_{2}$ and $S_{3}$ may now be considered functions of the independent variable $Z, \lambda^{\prime}\left(t_{n}\right)$ and $\lambda^{\prime \prime}\left(t_{n}\right)$. Thus, for each time $t_{n}, \lambda\left(t_{n}\right)$, $\lambda^{\prime}\left(t_{n}\right), \lambda^{\prime \prime}\left(t_{n}\right)$ and $\partial \lambda^{\prime \prime}\left(t_{n}\right) / \partial Z$ are found by solving a coupled system of non-linear ordinary differential equations (44). These results are then stored for use at $t_{n+1}$. The solution of (44), subject to appropriate boundary conditions at $\lambda=0$ and $i=h$, is obtained by the same procedure as was outlined for the initial elastic response.

Once the functions $\lambda(Z, t)$ and $\lambda^{\prime}(Z, t)$ have been determined, the current coordinates $x(Z, t)$ and $y(Z, t)$ are obtained directly from (6), while stresses are calculated from (29)(31), in which the integrals are approximated by the same procedure as was used in (37). 


\section{DISCUSSION}

All calculations are carried out for a slab in terms of the following non-dimensional quantities:

$$
\dot{Z}=\frac{Z}{H}, \quad \bar{h}=\frac{h}{H}, \quad \hat{i}=\frac{i}{H}, \quad i^{\prime \prime}=i^{\prime \prime} H .
$$

and

$$
\dot{\sigma}_{z:}=\frac{\sigma_{z:}}{C_{0}}, \quad \dot{\sigma}_{\mathrm{rr}}=\frac{\sigma_{\mathrm{rx}}}{C_{0}}, \quad \dot{\sigma}_{\mathrm{ru}}=\frac{\sigma_{3 y}}{C_{10}} .
$$

The parameter $\mu$ is chosen to be 0.1 , the ratio of long time to initial moduli $\gamma=C_{x} / C_{0}$ is picked to be 0.25 , and the relaxation time is assumed to be 1.0 . The initial condition (26), $\lambda^{\prime}(0, t)\left(=\lambda_{1}(0 . t)\right)$ is set as 1.1 , which is equivalent to saying $x(0, t)=X / \sqrt{1.1}=0.95 X$ and $y(0 . t)=Y / \sqrt{1.1}=0.95 Y$. For the problem where traction is prescribed, calculations are carried out for $\dot{\sigma}_{: z}(0,0,1, t)=0.2$, and for the problem where displacement is prescribed, $h(t)=1.2$. At each time step. the value of $\hat{\lambda}^{\prime \prime}\left(0, t_{n}\right)\left[=\hat{\lambda}_{2}\left(0, t_{n}\right)\right]$ is accepted as the final value when $|\hat{f}(1, t)-1.2|<\varepsilon$ for the problem where displacement is prescribed or $\left|\dot{\sigma}_{z:}(0,0, \mid, t)-0.2\right|<\varepsilon$ for the problem where traction is prescribed, where $8=5.0 \times 10^{-5}$. In general, for this choice of $\varepsilon$, about 10 iterations are required at each time step to arrive at an acceptable value for $\hat{\lambda}^{\prime \prime}(0, t)$. Time steps $t_{k}$ are chosen to vary logarithmically as did Wineman (1972). This permits small time increments for early times when quantities are undergoing large variations and larger time increments for later times when the variations are smaller. The time steps used are given by the relation $t_{k+1}=t_{k} \times 10^{4}$, where $A=0.2$ for $k=2,3, \ldots, 10, A=0.05$ for $k>11$, with $t_{z}=0.01$.

The response of the slab at $t=t_{1}=0$ to a step clongation, $\hat{\lambda}(1, t)=1.2$, for different $\lambda^{\prime}\left(1, t_{1}\right)$ [or different $C\left(t_{1}\right)$ is shown in Figs 1 and 2 . For example, given $\hat{\lambda}(1,0)=1.2$, while $C(0)$ is assumed to be $0.0,0.1$ and 0.3 , the values of $\lambda^{\prime}(1,0)$ are $1.315,1.27$ and 1.20 , respectively. It should be pointed out that there is no clear physical meaning for $C(t)$, though it is related to the boundary condition $\lambda^{\prime}(1, t)$. However, it is more convenient to

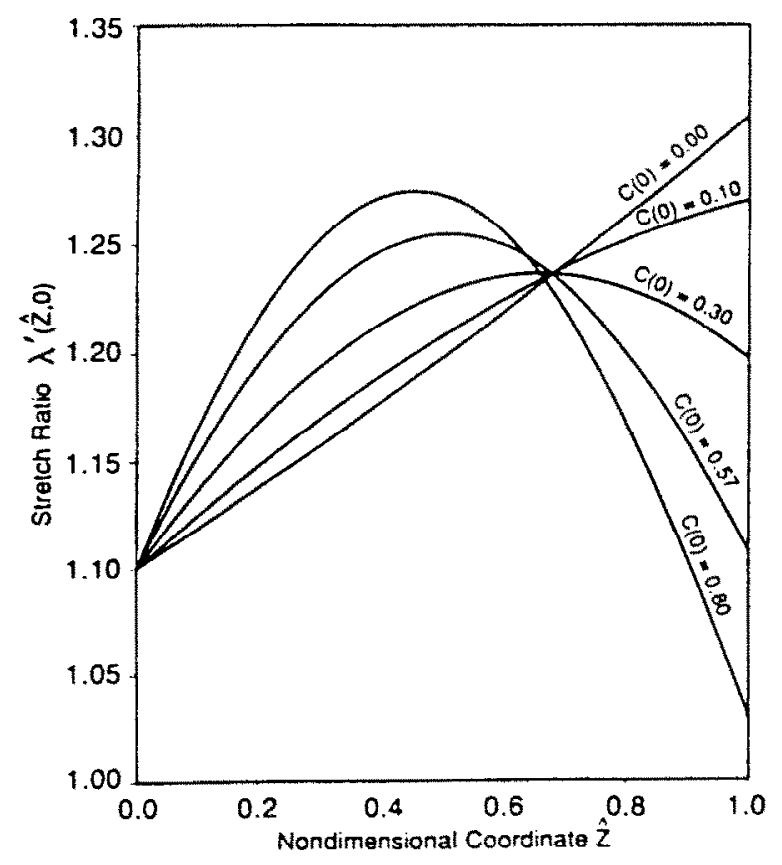

Fig. 1. Variation of the stretch ratio $:$ at initial time. 


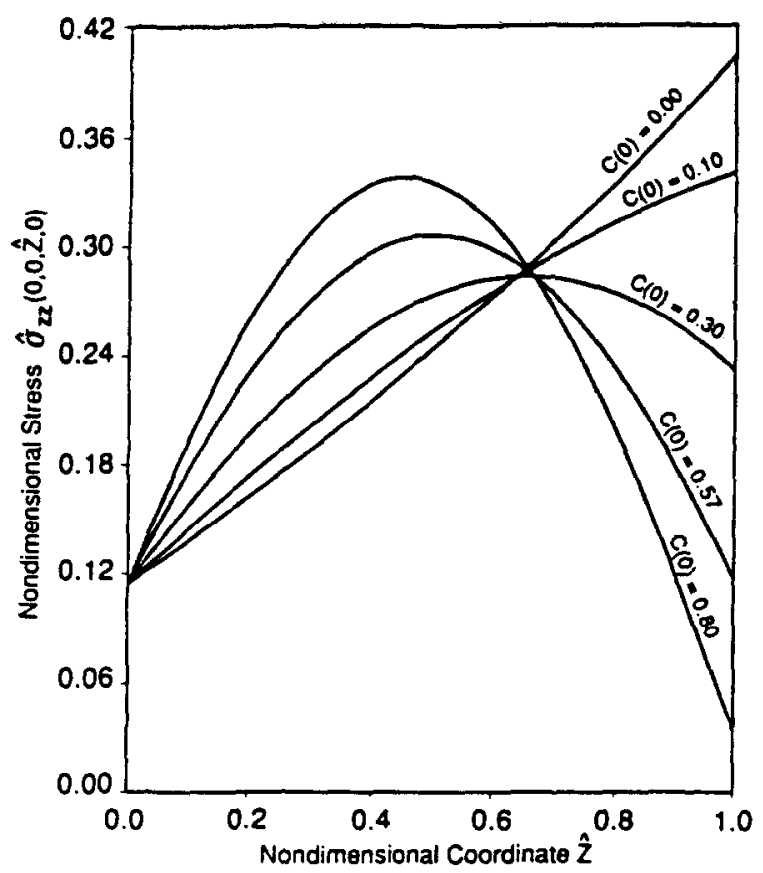

Fig. 2. Distribution of the stress $\sigma_{z:}$ at initial time.

deal with $C(t)$ instead of $\lambda^{\prime}(1, t)$ for computational convenience. Figure 2 shows how the stress in the slab $\dot{\sigma}_{::}(0,0, \dot{Z}, 0)$ varies with $C(0)$ [or $\left.\lambda^{\prime}(1,0)\right]$ at $t=0$. It can be seen from the figures that a symmetric deformation of the slab about the mid-plane is obtained by setting $C(0)=0.57$, which corresponds to $\lambda^{\prime}(1,0)=1.1$. It is interesting to note that if we still assume a constant value for $C(t)$ for $t>0$, for instance $C(t)=0.57$, though there is a symmetric distribution of the stretch ratio and stresses initially, as time progresses they become asymmetric as shown in Figs 3 and 4 . This implies that assigning a constant $C(t)$

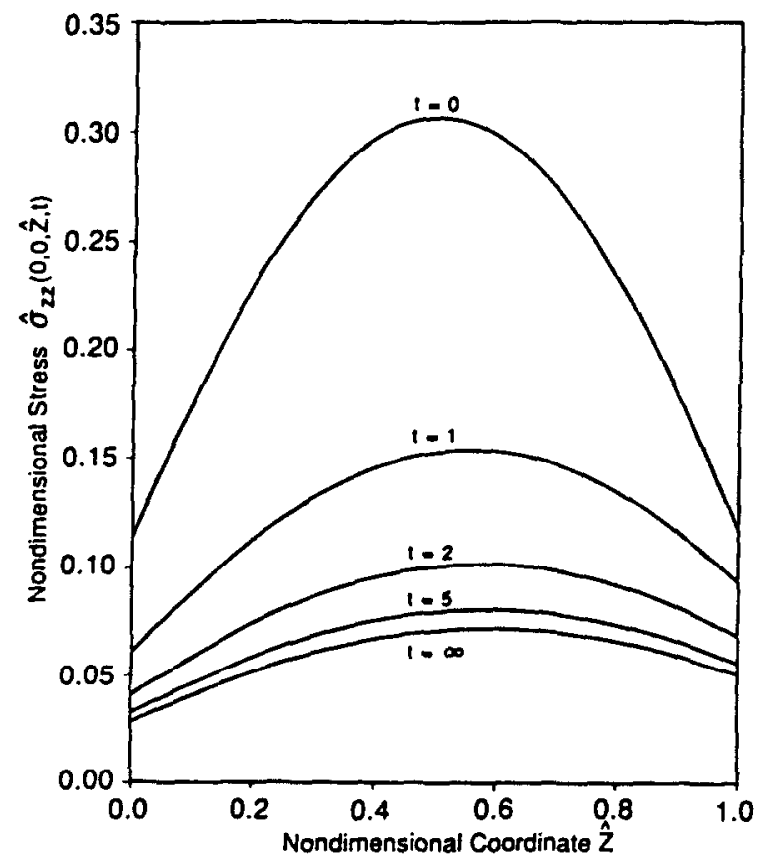

Fig. 3. Distribution of the stress $\sigma_{: \text {f }}$ for a constant value of $C(t)$ for the problem where displacement is specified. 


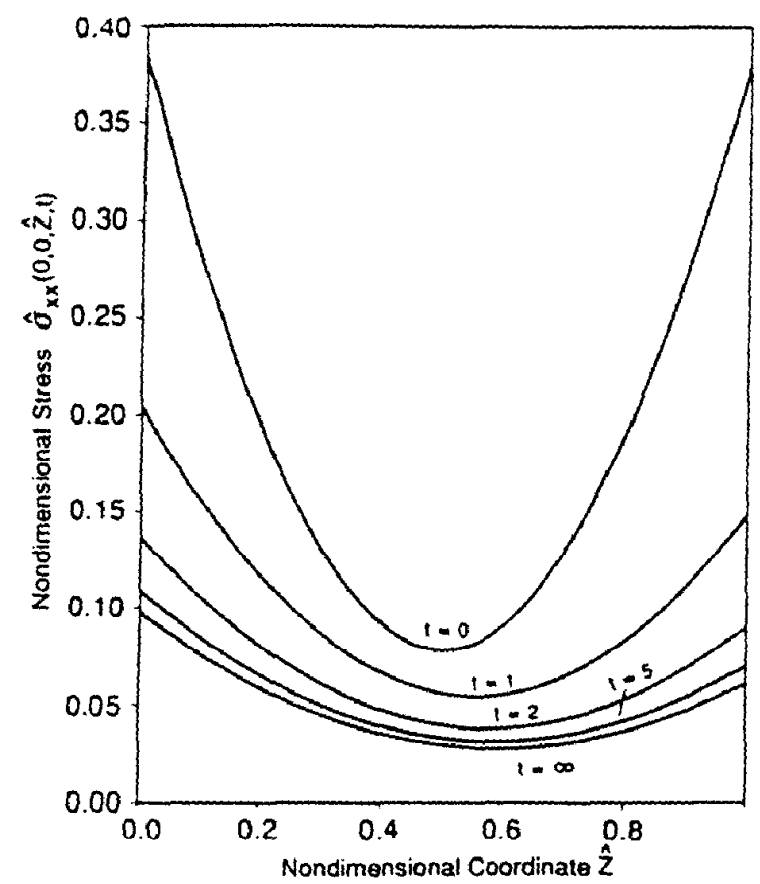

Fig. 4. Distribution of the stress $\sigma_{2}$, for a constant value of $($ (t) for the problem where displacement is specified.

corresponds to prescribing a time dependent $\lambda^{\prime}(1, t)$ [or $\left.x(t), y(t)\right]$ at the top plane of the viscoclastic slab as shown in Fig. 5 .

Generally, $C(t)$ is a function of $t$ as it appears in the governing equation (24). Motivated by the exponential decaying characteristic of the stresses with time, we assume that

$$
C(t)=\kappa_{1}+\kappa_{2}\left[1.0-\exp \left(-\frac{t}{\tau_{\mathrm{K}}}\right)\right]
$$

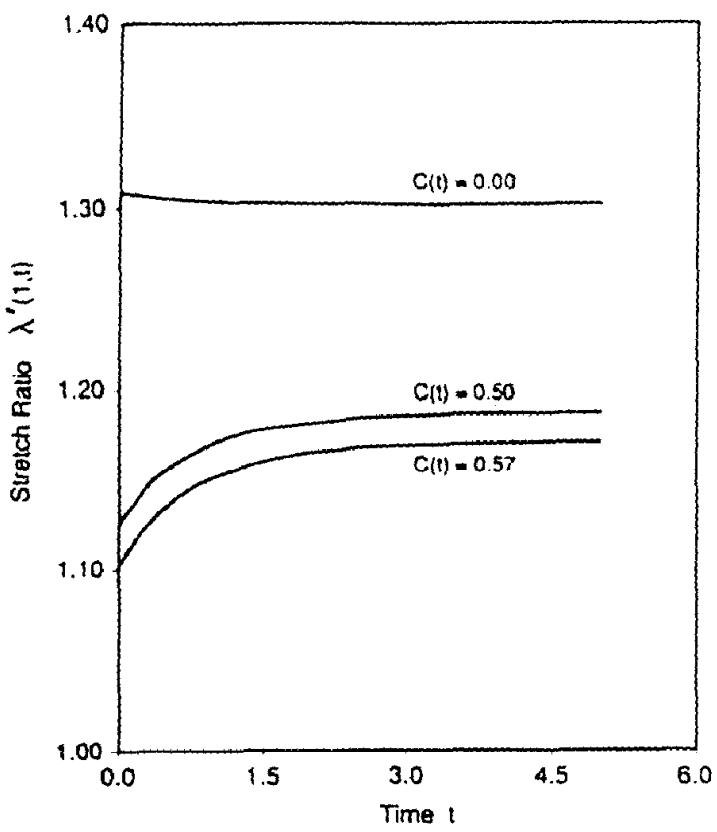

Fig. 5. Variation of the stretch ratio $2 \cdot(1, t)$ with time. 


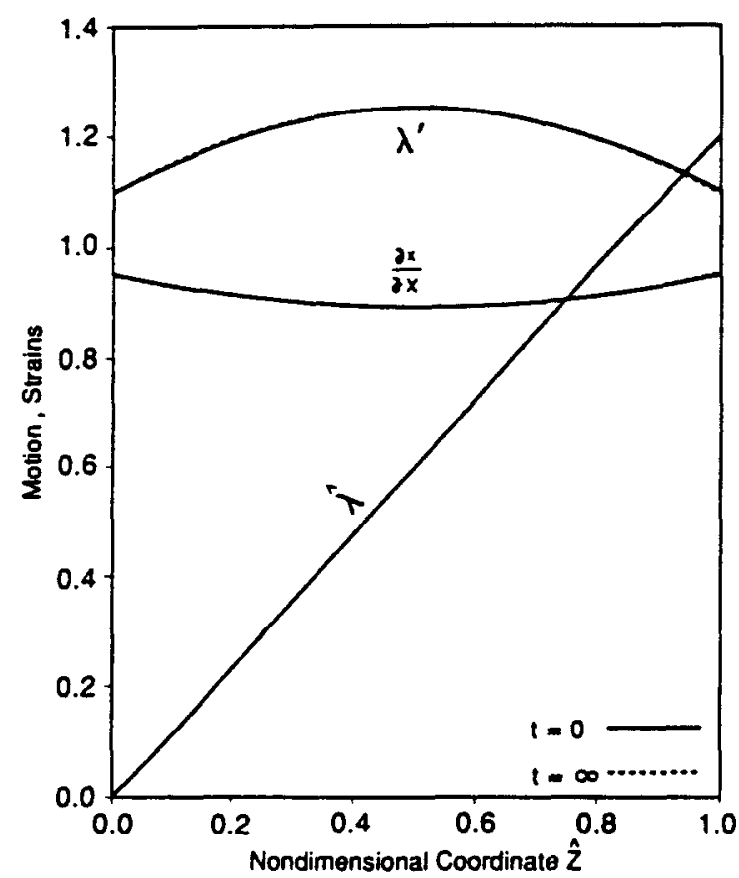

Fig. 6. Variation of the motion and strains when displacement is specified.

where $K_{1}$ and $K_{2}$ are constants. When $K_{1}=0.57$ and $K_{2}=0.30$, the stretch ratio $\lambda^{\prime}(\dot{Z}, t)$ and corresponding stresses $\hat{\sigma}_{: i}(0,0, \dot{Z}, t)$ and $\hat{\sigma}_{v}(0,0, \hat{Z}, t)$ relax symmetrically at all times as shown in Figs 6-8. It is also seen from these figures that there is very little deformation with time (see Fig. 6), which corresponds to the calse where a constant $\lambda^{\prime}(0,0)=\lambda^{\prime}(1,0)$ (note $x=X / \sqrt{\lambda^{\prime}}$ ) is assigned both at the bottom and top of the viscoelastic slab, respectively, and $\dot{\sigma}_{x,}(0,0,0, t)=\dot{\sigma}_{x,}(0,0,1, t)\left(\dot{\sigma}_{x t}=\dot{\sigma}_{y v}\right.$ from the original equations) for all time (sec Figs 7 and 8 ).

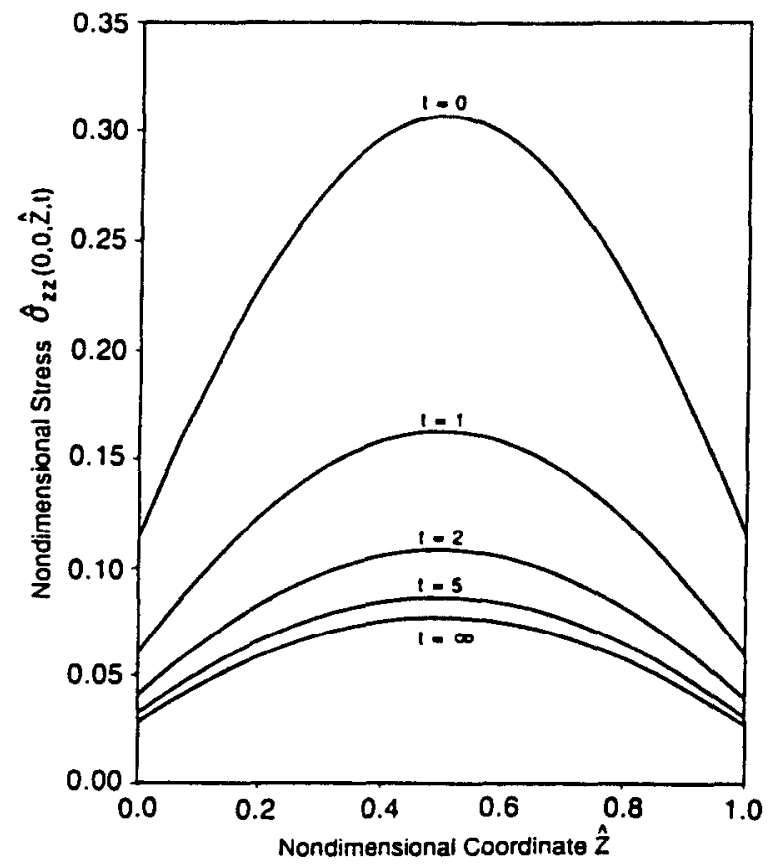

Fig. 7. Symmetric distribution of the stress $\sigma_{::}$at various times, when $C(t)$ is non-constant. 


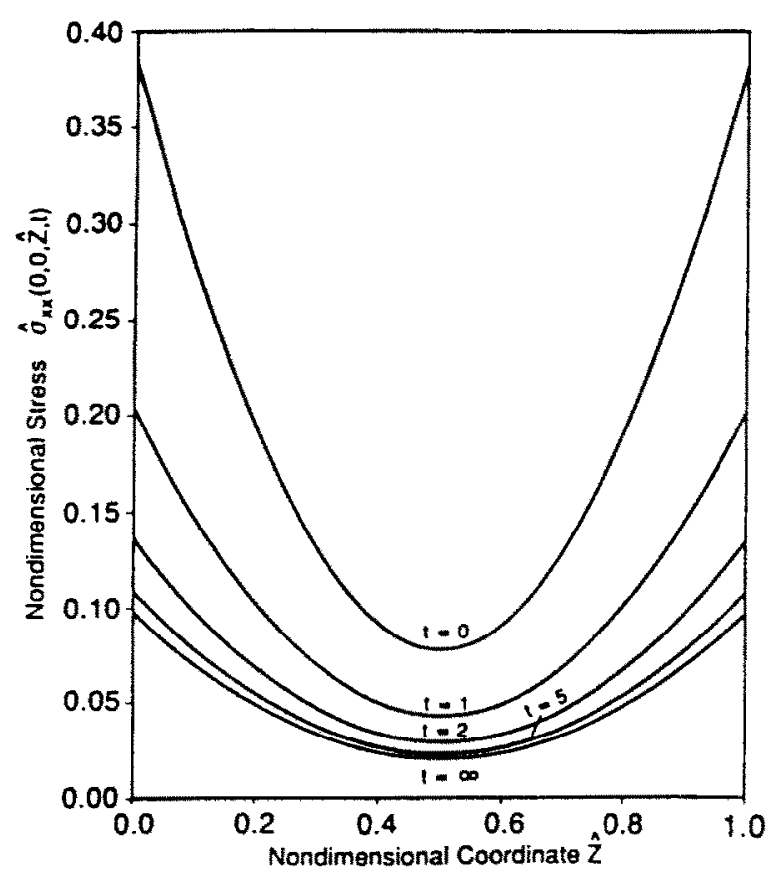

Fig. 8. Symmetric distribution of the stress $\dot{\sigma}_{n}$ at various times, when $C(n)$ is non-constant.

Having a clear idea about the relationship between $\lambda^{\prime}(1 . t)$ and $C(t)$, we now turn our attention to the non-uniform extension problem. Let us consider the case $C(t)=0.5 t \geqslant 0$, which corresponds to a time dependent deformation of the upper surface with $\lambda^{\prime}(1,0)=$ 1.12 as shown in Fig. 5. The numerical solutions for the case where $\lambda(1, t)=1.2$, $t \geqslant 0$, are represented in Figs $9-11$. The distributions of the kinematical quantities $\partial x / \partial X$ $(=\partial y / \partial Y=1.0 / \sqrt{\lambda}), \lambda^{\prime}$ and $\hat{\lambda}$ are shown in Fig. 9 for $t=0$ and $t \rightarrow \infty$. From the figure,

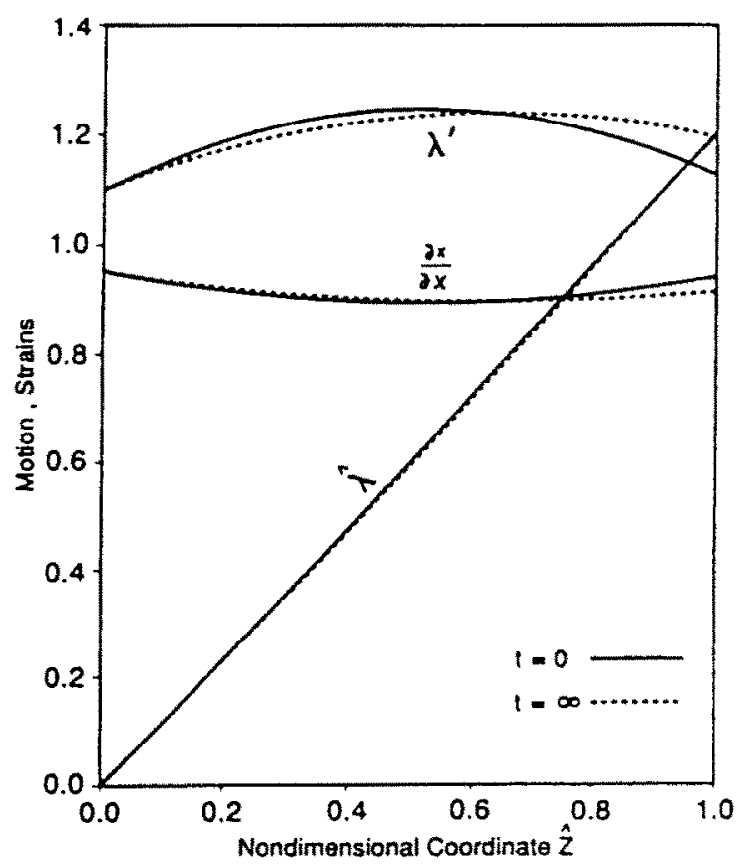

Fig. 9. Variation of the motion and strains when $C(t)=0.5$. 


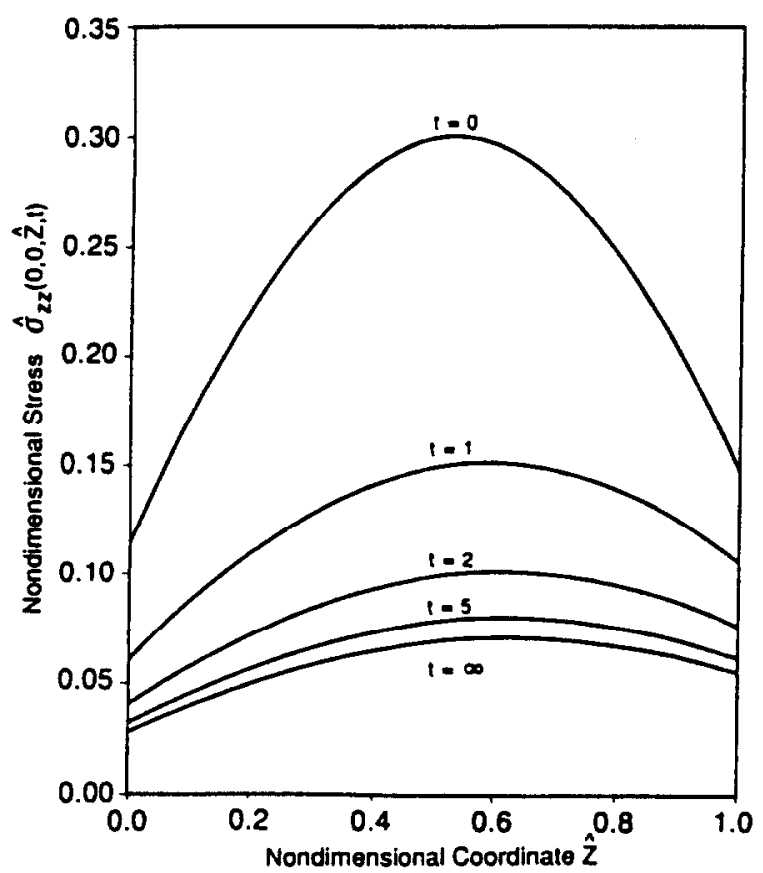

Fig. 10. Varriation of the stress $\dot{\sigma}_{i:}$ with time when $C(t)=0.5$.

it is clear that $\partial x / \partial X$ and $\lambda^{\prime}$ are non-uniform and $\lambda$ varies only slightly from its initial values but the variations in $\partial x / \partial X$ and $\lambda^{\prime}$ are relatively large, and are time dependent. The relaxation of the stresses $\dot{\sigma}_{z z}(0,0, \hat{Z}, t)$ and $\dot{\sigma}_{x x}(0,0, \dot{Z}, t)\left(=\dot{\sigma}_{v v}(0,0, \dot{Z}, t)\right)$ are evident from Figs 10 and 11. The stress $\dot{\sigma}_{z:}(0,0, \dot{Z}, \imath)$ of the lower boundary is smaller than that on the upper boundary, as $\lambda^{\prime}(0, t)$ is smaller than $\lambda^{\prime}(1, t)$. The same reason results in $\hat{\sigma}_{x x}(0,0,0, t)$ being larger than $\dot{\sigma}_{v x}(0,0,1, t)$.

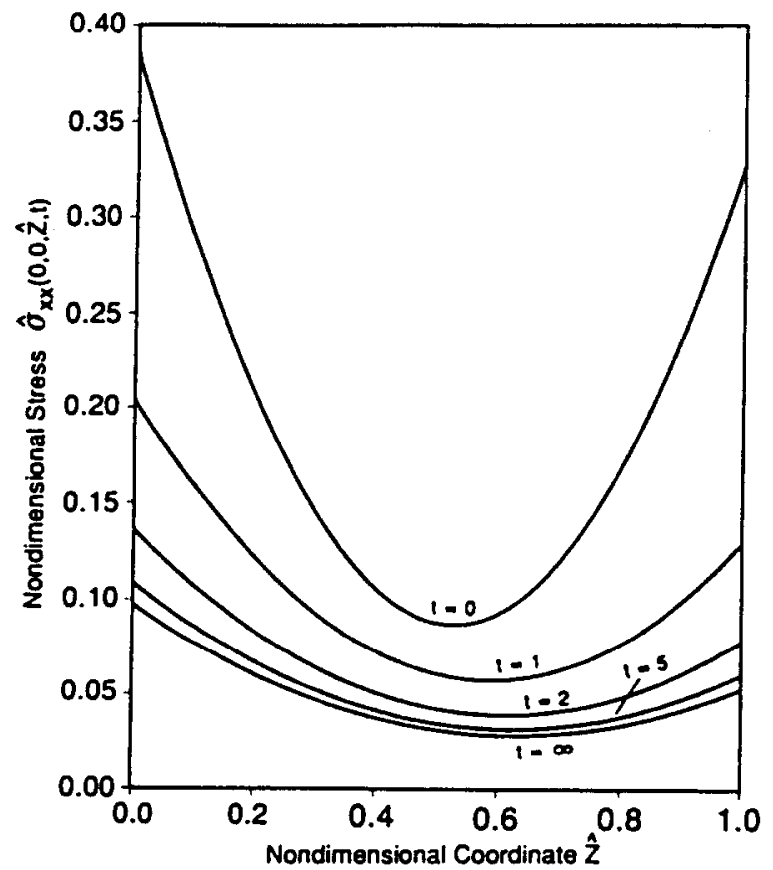

Fig. 11. Variation of the stress $\dot{\sigma}_{x s}$ with time when $C(t)=0.5$. 


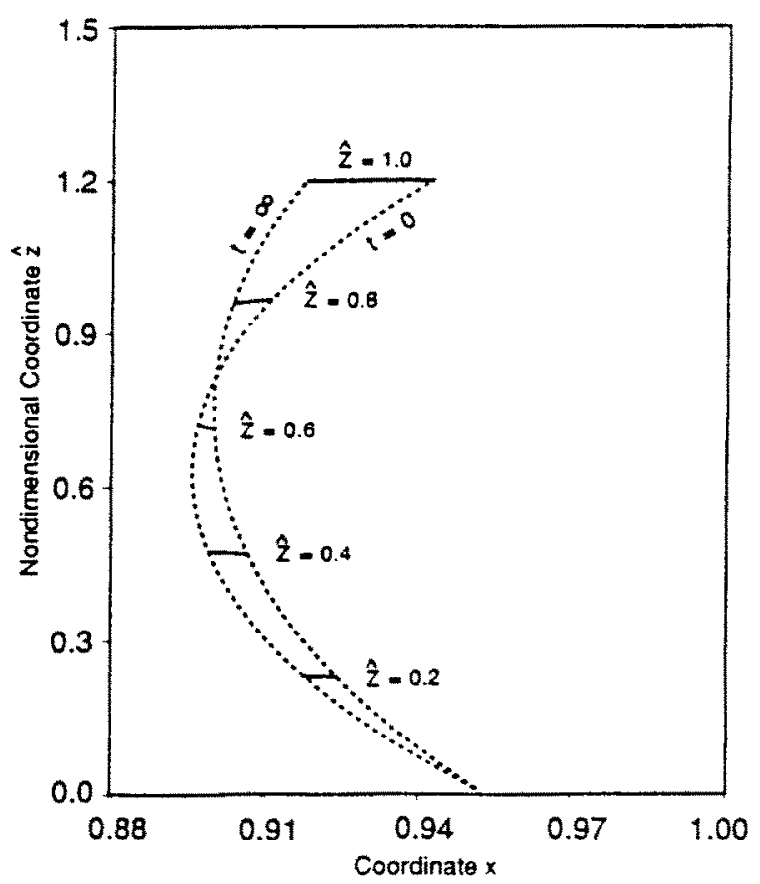

Fig. 12. Particle patths for the problem in which displacenent is specified

Figures 12 and 13 show the paths of particles originally at $X=1$ and $Y=0$ for the problems in which the displacement and traction are preseribed, respectively. In Figs 12 and 13 , the response at $t=0$ is the instantaneous clastic response. Also shown is the asymptotic viscoelastic response as $t \rightarrow \infty$. Thus, at any intermediate time the viscoelastic response is between the two limits shown in the figures.

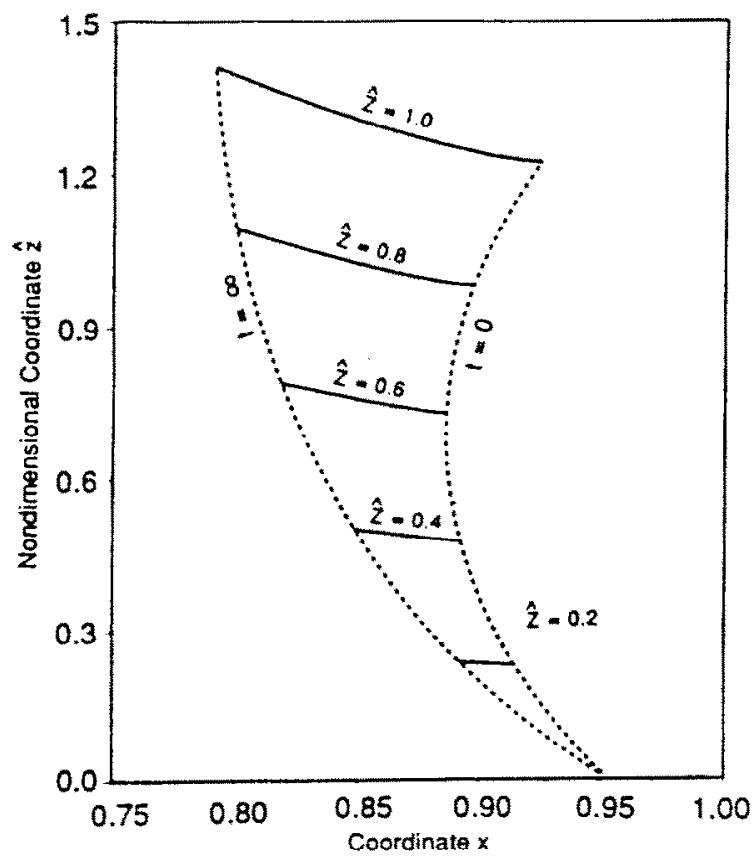

Fig. 13. Particle paths for the prothlem in which traction is prescribed. 


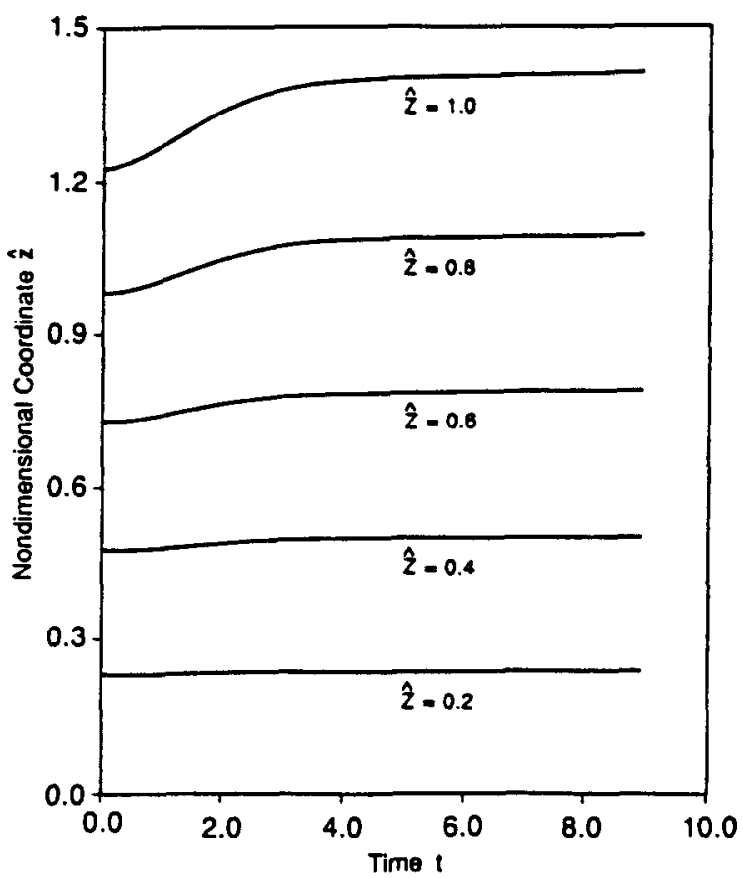

Fig. 14. Displacement histories for the problem in which traction is prescribed.

The response of the slab under a constant normal stress $\dot{\sigma}_{: z}(0,0,1, t)=\dot{\sigma}_{0}=0.2$ are shown in Figs 14-16. The deformation history of the slab for the problem is shown in Fig. 14. The displacement of the upper surface of the slab increases rapidly until $t=4.0$ and then slowly reaches a ceiling value as time increases. The displacement of the planes near the lower boundary, say $\hat{Z}=0.2$, however, increases slowly with time. The distributions of

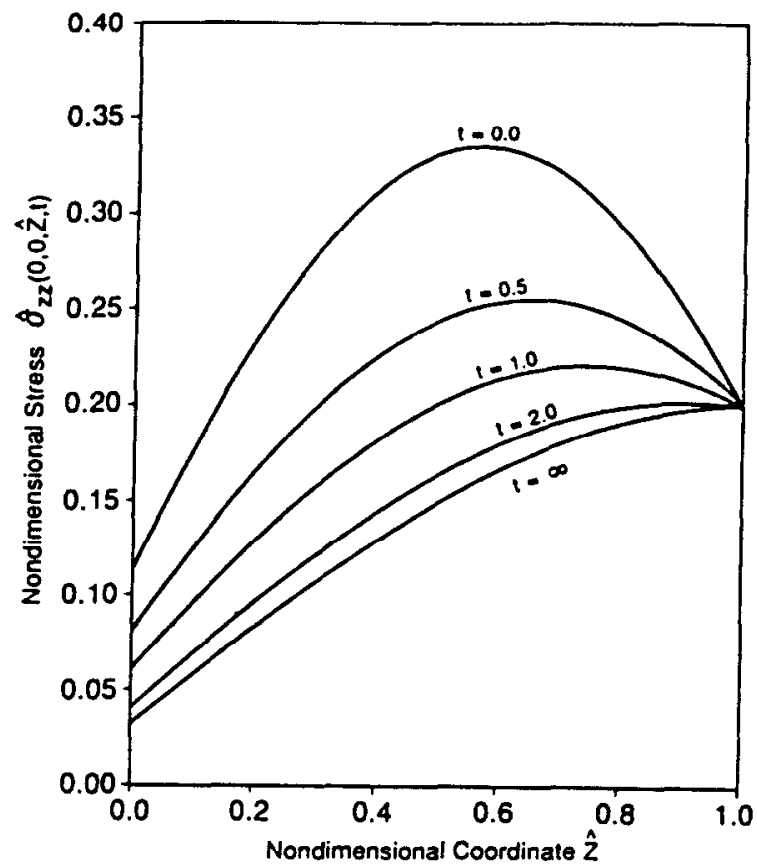

Fig. 15. Variation of the stress $\hat{\sigma}_{::}$with time when traction is prescribed. 


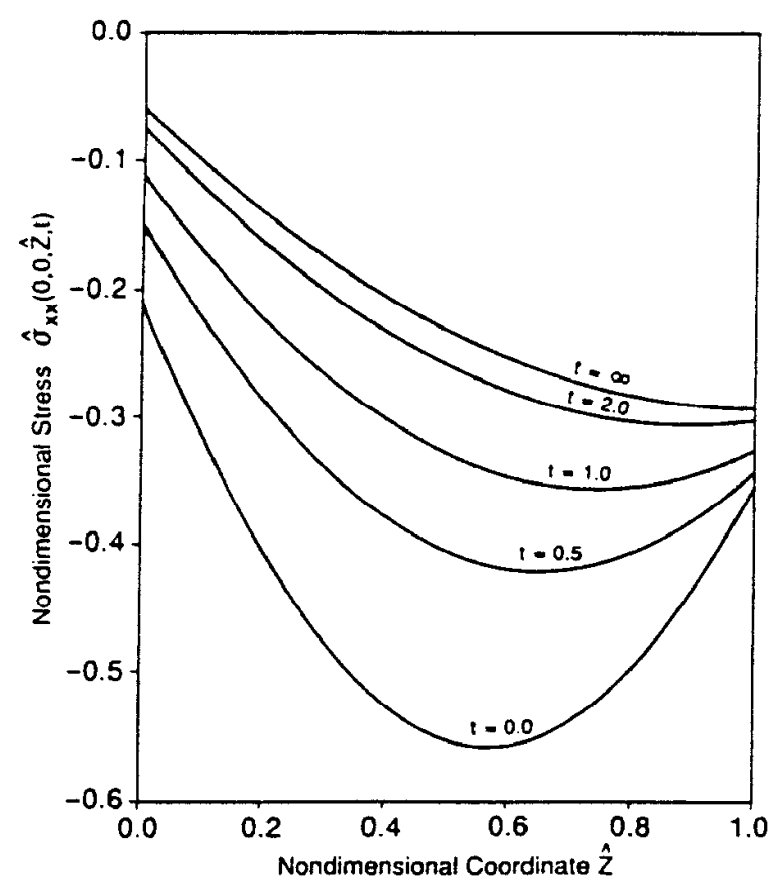

Fig. 16. Variation of the stress $\dot{\sigma}_{1}$, with time when traction is prescribed.

stresses $\dot{\sigma}_{::}(0,0, \hat{Z}, t)$ and $\dot{\sigma}_{u}\left(0,0, \dot{Z}_{,} t\right)$ through the slab are shown in Figs 15 and 16 , respectively. The stress $\dot{\sigma}_{x r}(0,0,1, t)\left[=\dot{\sigma}_{y y}(0,0,1, t)\right]$ changes with time. The distributions of the stresses $\dot{\sigma}_{: z}(0,0, \hat{Z}, t)$ and $\dot{\sigma}_{x x}(0,0, \hat{Z}, t)$ through the slab for the problems where displacement and traction are prescribed for the case where $C(t)=0.0$ (which corresponds to a time dependent $\lambda^{\prime}(1, t)$ with $\lambda^{\prime}(1,0)=1.315$ as shown in Fig. 5) are shown in Figs 1720. The stresses vary through the slab approximately linearly for both problems for the specific calse under consideration.

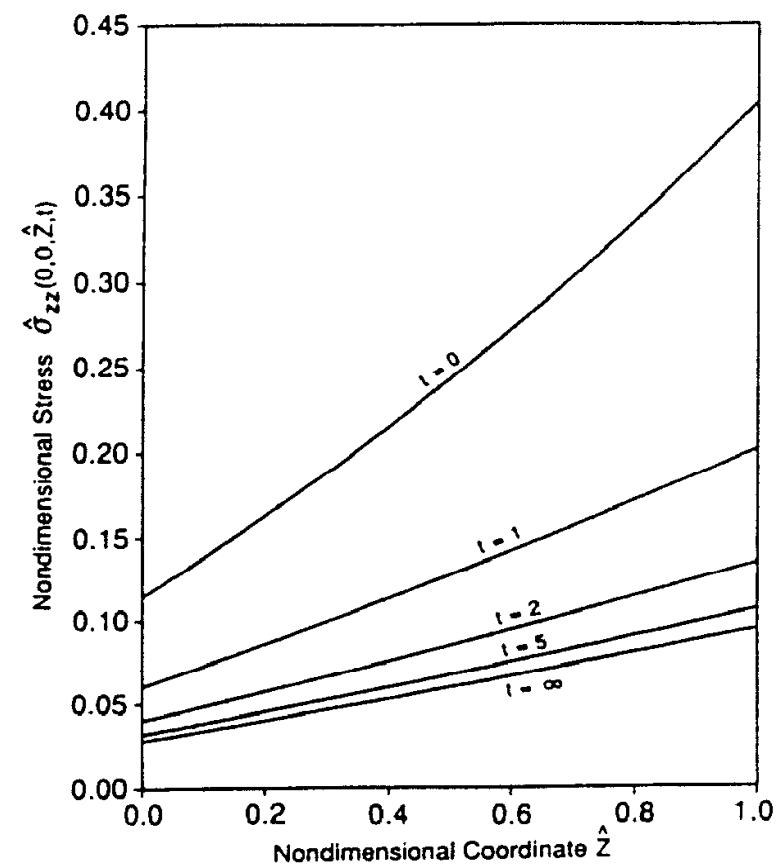

Fig. 17. Variation of the stress $\dot{\sigma}_{::}$with time for $C(t)=0$, when displacement is prescribed. 


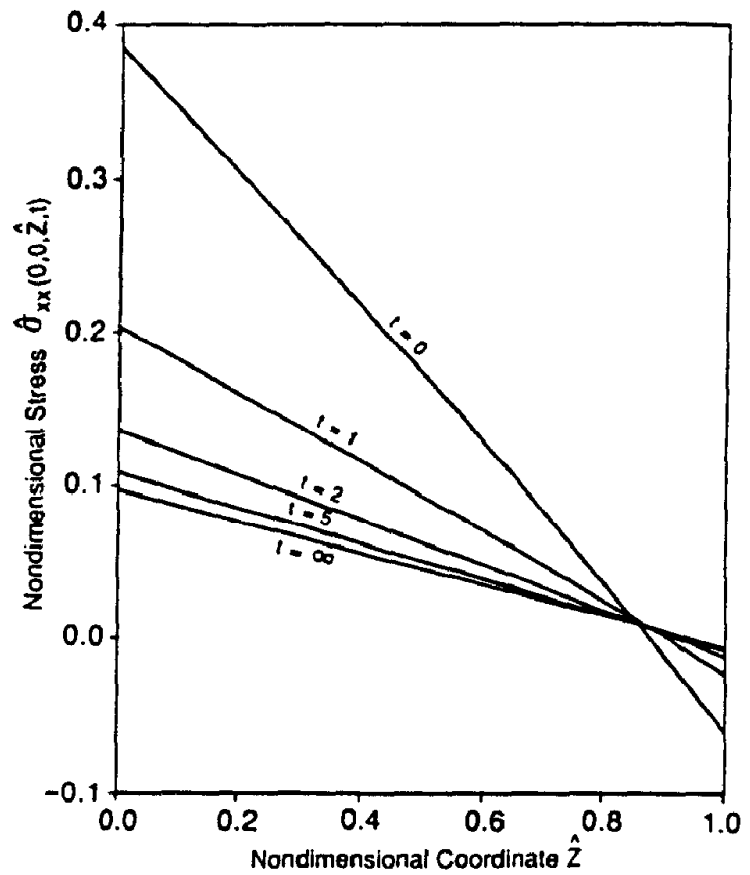

Fig. 18. Varration of the stress $\dot{\sigma}_{1}$, with tinte for $C(t)=0$, when displacement is prescribed.

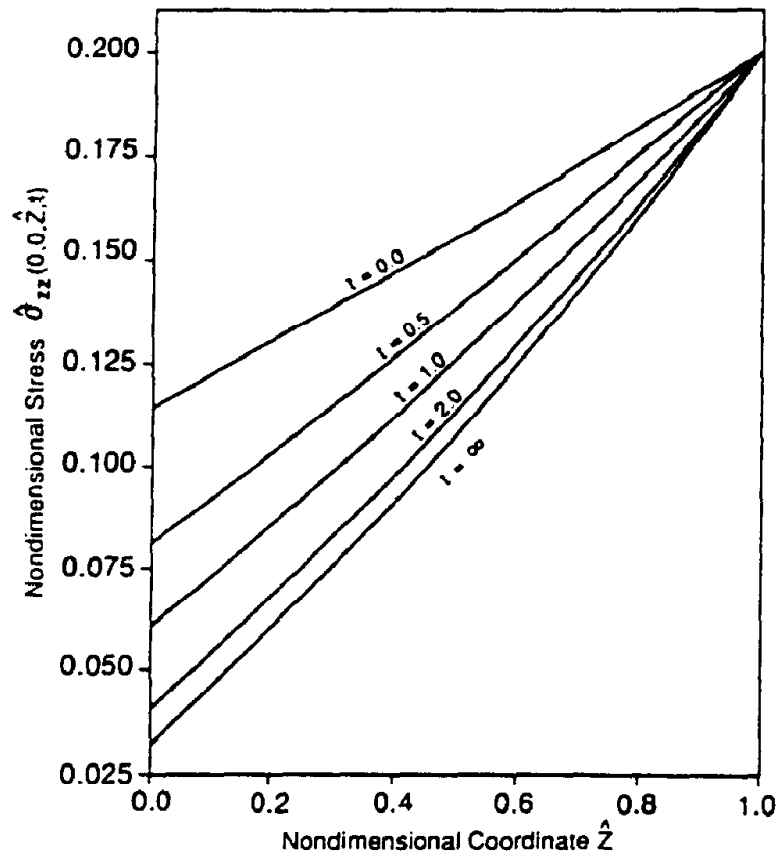

Fig. 19. Variation of the stress $\dot{\sigma}_{::}$with time for $C(t)=0$, when traction is prescribed. 


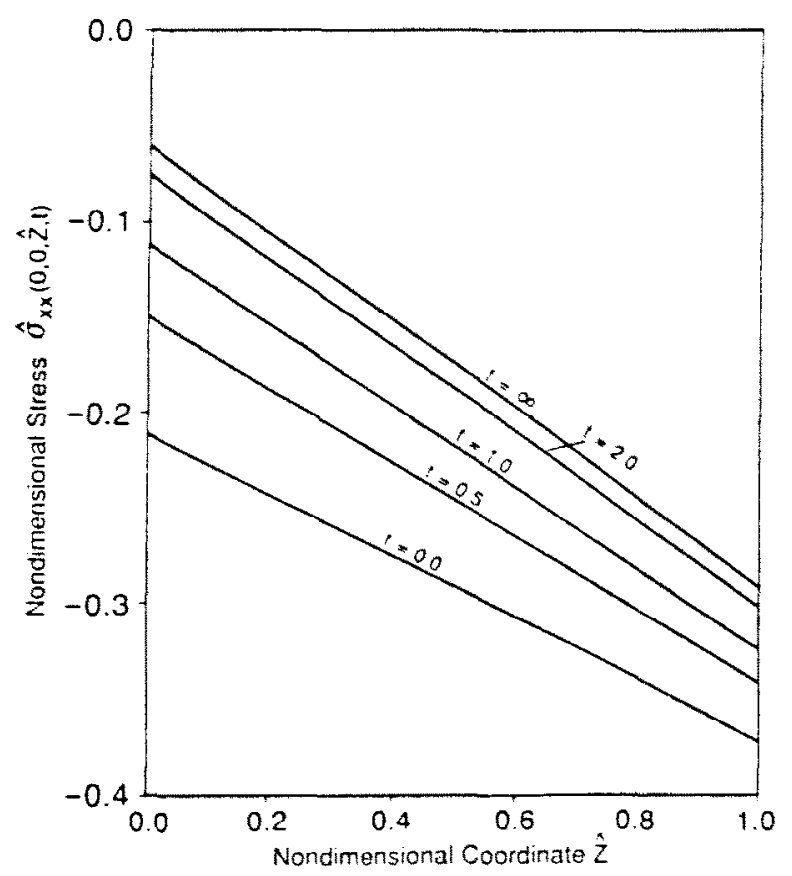

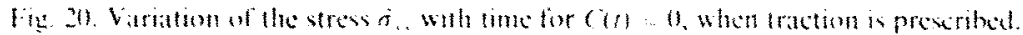

\section{RIITRINTIS}

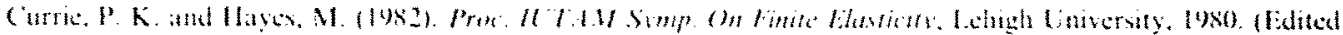

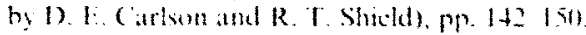

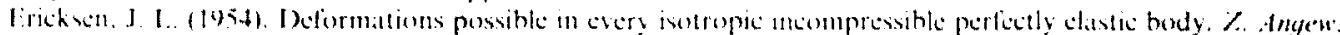
Wath. Imo. 5 , tho tho

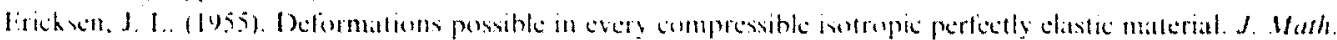
thix. 3. 126,128

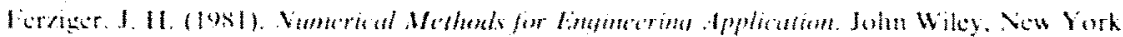

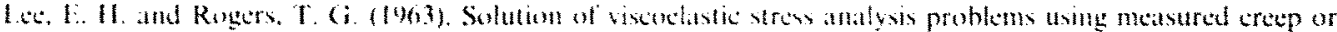

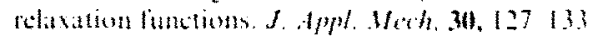

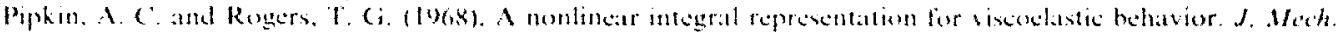
Plits. Solids I6. is 74 .

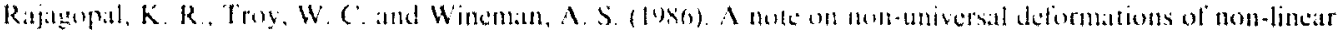

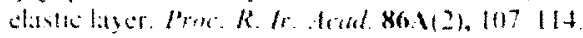

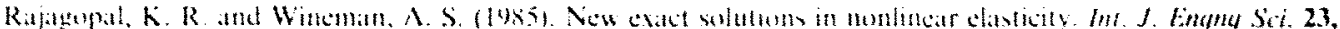
21723

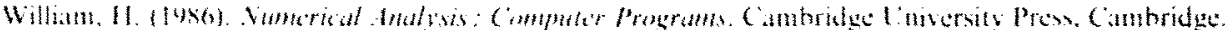

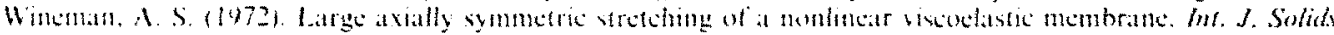
Siruturas 8,7757011

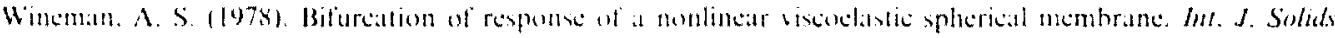
Sirmateris 14, 19? 212 